\title{
THE POISSON EQUATION IN HOMOGENEOUS SOBOLEV SPACES
}

\author{
TATIANA SAMROWSKI and WERNER VARNHORN
}

Received 9 August 2003

\begin{abstract}
We consider Poisson's equation in an $n$-dimensional exterior domain $G(n \geq 2)$ with a sufficiently smooth boundary. We prove that for external forces and boundary values given in certain $L^{q}(G)$-spaces there exists a solution in the homogeneous Sobolev space $S^{2, q}(G)$, containing functions being local in $L^{q}(G)$ and having second-order derivatives in $L^{q}(G)$. Concerning the uniqueness of this solution we prove that the corresponding nullspace has the dimension $n+1$, independent of $q$.
\end{abstract}

2000 Mathematics Subject Classification: 35A05, 35J05, 35J25.

1. Introduction. Let $G \subset \mathbb{R}^{n}(n \geq 2)$ be an exterior domain with a smooth boundary $\partial G$ of class $C^{2}$. We consider Poisson's equation concerning some scalar function $u$ :

$$
-\Delta u=f \quad \text { in } G, \quad u_{\mid \partial G}=\Phi .
$$

Here $f$ is given in $G$ and $\Phi$ is the boundary value prescribed on $\partial G$. As usual, $\Delta$ denotes the Laplacian in $\mathbb{R}^{n}$.

It is well known that in unbounded domains the treatment of differential equations causes special difficulties, and that the usual Sobolev spaces $W^{m, q}(G)$ are not adequate in this case: even for the Laplacian in $\mathbb{R}^{n}$ we find [4] that the operator $\Delta: W^{m, q}\left(\mathbb{R}^{n}\right) \rightarrow$ $W^{m-2, q}\left(\mathbb{R}^{n}\right)$ is not a Fredholm operator in general, as it is in the case of bounded domains. Thus in exterior domains, (1.1) have mostly been studied in connection with weight functions. Either (1.1) has been solved in weighted Sobolev spaces directly [8, $13,15]$, or it has first been multiplied by some weights and then solved in standard Sobolev spaces [20].

It is the aim of the present paper to prove the solvability of (1.1) in the homogeneous Sobolev spaces $S^{2, q}(G)(1<q<\infty)$ of the following type [5, 12]. Let $L^{q}(G)$ be the space of functions defined almost everywhere in $G$ such that the norm

$$
\|f\|_{q, G}:=\left(\int_{G}|f(x)|^{q} d x\right)^{1 / q}
$$

is finite. Then $S^{2, q}(G)$ is the space of all functions being local in $L^{q}(\bar{G})$ and having all second-order distributional derivatives in $L^{q}(G)$. We show that for $f \in L^{q}(G)$ and some boundary value $\Phi \in W^{2-1 / q, q}(\partial G)$ (see the notations below) there exists always a solution $u \in S^{2, q}(G)$. Concerning the uniqueness of this solution, we prove that the space of all $u \in S^{2, q}(G)$ satisfying (1.1) with $f=0$ and $\Phi=0$ has the dimension $n+1$, 
independent of $q$. This also holds for the case $n=2$. Similar results in slightly different spaces have been investigated by completely different methods in [17].

Throughout this paper, $G \subset \mathbb{R}^{n}(n \geq 2)$ is an exterior domain, that is, a domain whose complement is compact. Let $\bar{G}$ denote its closure in $\mathbb{R}^{n}$ and $\partial G$ its boundary, which we assume to be of class $C^{2}$ [1, page 67].

In the following, all function spaces contain real-valued functions. Let $D \subset \mathbb{R}^{n}$ be any domain with a compact boundary $\partial D$ of class $C^{2}$, or let $D=\mathbb{R}^{n}$. Besides the spaces $L^{q}(D)$, we need the well-known functions spaces $C^{\infty}(D), C_{0}^{\infty}(D)$, and the space $C_{0}^{\infty}(\bar{D})$, containing the restrictions $f_{\mid \bar{D}}$ of functions $f \in C_{0}^{\infty}\left(\mathbb{R}^{n}\right)$.

We call a function $u$ local in $L^{q}(\bar{D})(1<q<\infty)$ and write $u \in L_{\text {loc }}^{q}(\bar{D})$ if $u \in L^{q}(D \cap B)$ for every open ball $B \subset \mathbb{R}^{n}$. Note that this space does not coincide with the usual space $L_{\text {loc }}^{q}(D)$ in general (except for $\left.D=\mathbb{R}^{n}\right)$. For $D \neq \mathbb{R}^{n}$ we find $L^{q}(D) \subset L_{\text {loc }}^{q}(\bar{D}) \subset L_{\text {loc }}^{q}(D)$ and, if $D$ is bounded, $L^{q}(D)=L_{\mathrm{loc}}^{q}(\bar{D})$ and $L^{q}(D) \subset L_{\mathrm{loc}}^{q}(D)$.

By $W^{m, q}(D)\left(m=0,1,2 ; W^{0, q}(D)=L^{q}(D)\right)$ we mean the usual Sobolev space of functions $u$ such that $D^{\alpha} u \in L^{q}(D)$ for all multi-indices $\alpha=\left(\alpha_{1}, \ldots, \alpha_{n}\right) \in \mathbb{N}_{0}^{n}=\{0,1, \ldots\}^{n}$ with $|\alpha|:=\alpha_{1}+\cdots+\alpha_{n} \leq m[1]$. Here we used

$$
D^{\alpha} u=D_{1}^{\alpha_{1}} D_{2}^{\alpha_{2}} \cdots D_{n}^{\alpha_{n}} u, \quad D_{i}=\frac{\partial}{\partial x_{i}} \quad\left(i=1, \ldots, n ; x=\left(x_{1}, \ldots, x_{n}\right) \in \mathbb{R}^{n}\right) .
$$

The spaces $W_{\mathrm{loc}}^{m, q}(D)$ and $W_{\mathrm{loc}}^{m, q}(\bar{D})$ are defined analogously.

We need the fractional-order space $W^{2-1 / q, q}(\partial D)$, which contains the trace $u_{\mid \partial D}$ of all $u \in W_{\text {loc }}^{2, q}\left(\mathbb{R}^{n}\right)$ [1, page 216]. The norm in $W^{2-1 / q, q}(\partial D)$ is denoted by $\|\cdot\|_{2-1 / q, q, \partial D}$.

The term $\nabla u=\left(D_{j} u\right)_{j=1, \ldots, n}$ is the gradient of $u$ and $\nabla^{2} u=\left(D_{i} D_{j} u\right)_{i, j=1, \ldots, n}$ means the system of all second-order derivatives of $u$. For these terms we define the seminorms

$$
\|\nabla u\|_{q, D}:=\left(\sum_{k=1}^{n}\left\|D_{k} u\right\|_{q, D}^{q}\right)^{1 / q}, \quad\left\|\nabla^{2} u\right\|_{q, D}:=\left(\sum_{j, k=1}^{n}\left\|D_{j} D_{k} u\right\|_{q, D}^{q}\right)^{1 / q},
$$

and introduce for $m=1,2$ and $1<q<\infty$ the homogeneous Sobolev spaces

$$
S^{m, q}(D)=\left\{u \in L_{\mathrm{loc}}^{q}(\bar{D}) \mid\left\|\nabla^{m} u\right\|_{q, D}<\infty\right\}
$$

Finally, concerning the norms and seminorms, we sometimes omit the domain of definition if it is obvious and use $\|\cdot\|_{q}$ or $\|\cdot\|_{2-1 / q, q}$ instead of $\|\cdot\|_{q, G}$ or $\|\cdot\|_{2-1 / q, q, \partial G}$, for example.

2. Potential theory. Besides the Poisson equation (1.1) we also consider the special case of Laplace's equation with Dirichlet boundary condition

$$
-\Delta u=0 \quad \text { in } G, \quad u_{\mid \partial G}=\Phi
$$

These equations have mostly been studied with methods of potential theory (see, e.g., $[9,18])$. We collect some well-known facts in this section. 
Let $E_{n}(n \geq 2)$ in the following denote the fundamental solution of the Laplacian such that $-\Delta E_{n}(x)=\delta(x)$, where $\delta$ is Dirac's distribution in $\mathbb{R}^{n}$. It is well known that

$$
E_{2}(x)=-\frac{\ln |x|}{\omega_{2}}, \quad E_{n}(x)=\frac{|x|^{2-n}}{(n-2) \omega_{n}} \quad(n \geq 3),
$$

where $\omega_{n}$ is the area of the $(n-1)$-dimensional unit sphere in $\mathbb{R}^{n}(n \geq 2)$.

LEMMA 2.1. Let $G \subset \mathbb{R}^{n}(n \geq 2)$ be an exterior domain with boundary $\partial G$ of class $C^{2}$, and let $a \in \mathbb{R}$ and $\Phi \in C(\partial G)$ be given. Then there is at most one $u \in C^{\infty}(G) \cap C(\bar{G})$ satisfying (2.1) in $G$, if we require in addition for $|x| \rightarrow \infty$ :

$$
\begin{array}{ccc}
u(x)-a \ln |x|=O(1) \quad(n=2), & u(x)=O\left(|x|^{2-n}\right) \quad(n \geq 3), \\
\nabla^{m} u(x)=O\left(|x|^{2-n-m}\right) & (n \geq 2 ; m=1,2) .
\end{array}
$$

Proof. Let $u=u^{1}-u^{2}$ be the difference of two solutions $u^{1}$ and $u^{2}$ with the required decay properties above. Define the bounded domain $G_{r}=G \cap B_{r}(O)$, where $B_{r}(O) \subset \mathbb{R}^{n}$ denotes an open ball with center at zero and radius $r$ such that $\partial G \subset B_{r}(O)$. Thus in $G_{r}$ we may apply Green's first identity, obtaining

$$
\int_{G_{r}}|\nabla u|^{2} d x=\int_{\partial B_{r}}\left(\partial_{N} u\right) u d o,
$$

because the boundary integral over $\partial G$ vanishes. Here $N$ denotes the outward (with respect to $G_{r}$ ) unit normal vector on the boundary $\partial B_{r}=\partial B_{r}(O)$ and $\partial_{N} u$ is the normal derivative of $u$. Now due to the decay properties of $u$, the right-hand side in (2.5) tends to zero as $r \rightarrow \infty$. This is obvious if $n \geq 3$. For $n=2$, using the expansion theorem for harmonic functions at infinity [18, page 523], we find $u(x)=O(1)$ and $\nabla u(x)=$ $O\left(|x|^{-2}\right)$ as $|x| \rightarrow \infty$, which implies the assertion above, too. It follows that $\nabla u=$ 0 in $G$, hence $u=0$ in $G$ because $u$ vanishes on the boundary $\partial G$. This proves the uniqueness.

To show the existence of a solution with the required decay properties, we use the boundary integral equations' method. We define the single-layer potential

$$
\left(E^{n} \Theta\right)(x)=\int_{\partial G} E_{n}(x-y) \Theta(y) d o_{y} \quad(x \notin \partial G),
$$

the double-layer potential

$$
\left(D^{n} \Theta\right)(x)=-\int_{\partial G} \partial_{N(y)} E_{n}(x-y) \Theta(y) d o_{y} \quad(x \notin \partial G),
$$

and the normal derivative of the single-layer potential

$$
\left(H^{n} \Theta\right)(x)=-\int_{\partial G} \partial_{N(x)} E_{n}(x-y) \Theta(y) d o_{y} \quad(x \notin \partial G) .
$$

Here and in the following, $N=N(z)$ is the outward (with respect to the bounded domain $\left.G_{b}=\mathbb{R}^{n} \backslash \bar{G}\right)$ unit normal vector in $z \in \partial G$, and $\Theta \in C(\partial G)$ is the unknown source density. 
Then we have the continuity relation

$$
\left(E^{n} \Theta\right)^{e}=\left(E^{n} \Theta\right)^{i}=E^{n} \Theta \quad \text { on } \partial G
$$

and, due to the regularity of the boundary, the jump relations

$$
\begin{aligned}
& D^{n} \Theta-\left(D^{n} \Theta\right)^{e}=\left(D^{n} \Theta\right)^{i}-D^{n} \Theta=\frac{1}{2} \Theta \text { on } \partial G, \\
& H^{n} \Theta-\left(H^{n} \Theta\right)^{e}=\left(H^{n} \Theta\right)^{i}-H^{n} \Theta=-\frac{1}{2} \Theta \text { on } \partial G .
\end{aligned}
$$

Here the indices $e$ and $i$ stand for the limits from the exterior domain $G$ and the interior domain $G_{b}:=\mathbb{R}^{n} \backslash \bar{G}$, respectively.

Now we first assume $n \geq 3$. Following [3, 11] (here for the case of Helmholtz's equation), for the solution of (2.1) we choose in $G$ the mixed ansatz

$$
u=D^{n} \Theta-\alpha E^{n} \Theta \quad(0<\alpha \in \mathbb{R})
$$

consisting of a double- and a single-layer potential. Then by means of (2.9) and (2.10), we obtain the second-kind Fredholm boundary integral equation

$$
\Phi=-\frac{1}{2} \Theta+D^{n} \Theta-\alpha E^{n} \Theta \quad \text { on } \partial G
$$

for the unknown source density $\Theta \in C(\partial G)$. To see that (2.13) is uniquely solvable for all boundary values $\Phi \in C(\partial G)$, let $0 \neq \Psi$ be a solution of the homogeneous adjoint integral equation

$$
0=-\frac{1}{2} \Psi+H^{n} \Psi-\alpha E^{n} \Psi \quad \text { on } \partial G
$$

By (2.9) and (2.11), this implies $\alpha\left(E^{n} \Psi\right)^{i}=\left(H^{n} \Psi\right)^{i}=-\left(\partial_{N} E^{n} \Psi\right)^{i}$, and Green's first identity yields

$$
\int_{G_{b}}\left|\nabla\left(E^{n} \Psi\right)\right|^{2} d x=\int_{\partial G}\left(E^{n} \Psi\right)^{i}\left(\partial_{N} E^{n} \Psi\right)^{i} d o=-\alpha \int_{\partial G}\left|E^{n} \Psi\right|^{2} d o,
$$

hence $E^{n} \Psi=0$ in $\bar{G}_{b}$. This implies $\left(E^{n} \Psi\right)^{e}=0$ using (2.9), and the uniqueness statement above yields $E^{n} \Psi=0$ in $G$, too. Thus $E^{n} \Psi=0$ in the whole $\mathbb{R}^{n}$, which implies $H^{n} \Psi=0$ in $G$ and in $G_{b}$, and we obtain $\Psi=0$ by (2.11), as asserted. This proves the existence in the case $n \geq 3$ by the Fredholm alternative theorem.

Now let $n=2$. As in [10] (for the case of Stokes' equations) we use in $G$ the ansatz

$$
u=-a \frac{\omega_{2}}{|\partial G|} E^{2} 1+D^{2} \Theta-\alpha E^{2} M \Theta-\beta \Theta_{M} \quad(0<\alpha \in \mathbb{R}, 0 \neq \beta \in \mathbb{R}) .
$$


Here $a \in \mathbb{R}$ is the prescribed constant from (2.3), $|\partial G|:=\int_{\partial G} d o$ is the surface area, $E^{2} 1$ is the single-layer potential with constant density $\Psi=1$, and the projector $M$ is defined by

$$
\Theta \longrightarrow M \Theta:=\Theta-\Theta_{M}
$$

with the surface mean value

$$
\Theta_{M}:=\frac{1}{|\partial G|} \int_{\partial G} \Theta(y) d o
$$

which implies

$$
(M \Theta)_{M}=\frac{1}{|\partial G|} \int_{\partial G}\left(\Theta(y)-\Theta_{M}\right) d o=\Theta_{M}-\Theta_{M}=0
$$

This ansatz indeed satisfies the prescribed decay condition $u(x)-a \ln |x|=O(1)$ as $|x| \rightarrow \infty$, which can be seen as follows:

$$
\begin{aligned}
-a \frac{\omega_{2}}{|\partial G|} E^{2} 1(x) & =a \frac{1}{|\partial G|} \int_{\partial G} \ln |x-y| d o_{y} \\
& =a \ln |x|+a \frac{1}{|\partial G|} \int_{\partial G} \ln \frac{|x-y|}{|x|} d o_{y} \\
& =a \ln |x|+o(1) \quad \text { as }|x| \longrightarrow \infty
\end{aligned}
$$

For the other terms, we find

$$
\begin{gathered}
D^{2} \Theta(x)=\int_{\partial G} \frac{(x-y) \cdot N(y)}{\omega_{2}|x-y|^{2}} \Theta(y) d o_{y}=O\left(|x|^{-1}\right), \\
E^{2} M \Theta(x)=\frac{1}{\omega_{2}} \int_{\partial G} \ln \frac{1}{|x-y|}(M \Theta)(y) d o_{y}+\frac{1}{\omega_{2}} \ln |x| \int_{\partial G}(M \Theta)(y) d o_{y} d o_{y} \\
=\frac{1}{\omega_{2}} \int_{\partial G} \ln \frac{|x|}{|x-y|}(M \Theta)(y) d o_{y}=o(1),
\end{gathered}
$$

and finally $\Theta_{M}=O(1)$ as $|x| \rightarrow \infty$, which implies the required decay condition (2.3).

Now using (2.9) and (2.10) again, we obtain the second-kind Fredholm boundary integral equation

$$
\Phi+\frac{a \omega_{2}}{|\partial G|} E^{2} 1=-\frac{1}{2} \Theta+D^{2} \Theta-\alpha E^{2} M \Theta-\beta \Theta_{M} \quad \text { on } \partial G
$$

To see that (2.22) has a unique solution $\Theta \in C(\partial G)$ for all boundary values $\Phi \in C(\partial G)$ and all $a \in \mathbb{R}$, let $0 \neq \Psi$ solve the homogeneous adjoint integral equation

$$
0=-\frac{1}{2} \Psi+H^{2} \Psi-\alpha M E^{2} \Psi-\beta \Psi_{M} \quad \text { on } \partial G
$$


Because for any constant $c \in \mathbb{R}$ we have $-(1 / 2) c+D^{2} c=0[18$, page 511$]$ and $E^{2} M c=0$, we find

$$
\begin{aligned}
0 & =\left\langle c,-\frac{1}{2} \Psi+H^{2} \Psi-\alpha M E^{2} \Psi-\beta \Psi_{M}\right\rangle \\
& =\left\langle-\frac{1}{2} c+D^{2} c-\alpha E^{2} M c, \Psi\right\rangle-\beta\left\langle c, \Psi_{M}\right\rangle \\
& =-\beta\left\langle c, \Psi_{M}\right\rangle,
\end{aligned}
$$

where here

$$
\langle\psi, \phi\rangle:=\int_{\partial G} \psi(y) \phi(y) d o
$$

denotes the corresponding duality. It follows that $\Psi_{M}=0$ and $M \Psi=\Psi$, hence $\Psi$ is a solution of

$$
0=-\frac{1}{2} \Psi+H^{2} \Psi-\alpha M E^{2} \Psi \quad \text { on } \partial G
$$

too. Using (2.11), this implies

$$
\left(H^{2} \Psi\right)^{i}=-\frac{1}{2} \Psi+H^{2} \Psi=\alpha M E^{2} \Psi \quad \text { on } \partial G,
$$

and from Green's first identity, we obtain

$$
\begin{aligned}
\int_{G_{b}}\left|\nabla E^{2} \Psi\right| d x & =\int_{\partial G} E^{2} \Psi \cdot \partial_{n} E^{2} \Psi d o=-\int_{\partial G} E^{2} \Psi \cdot\left(H^{2} \Psi\right)^{i} d o \\
& =-\alpha \int_{\partial G} E^{2} \Psi \cdot M E^{2} \Psi d o=-\alpha \int_{\partial G}\left|M E^{2} \Psi\right|^{2} d o .
\end{aligned}
$$

Since $\alpha>0$, it follows that $M E^{2} \Psi=0$ on $\partial G$, which means $E^{2} \Psi=\left(E^{2} \Psi\right)_{M}=$ const. on $\partial G$. By Lemma 2.1, this implies $E^{2} \Psi=$ const. in $G_{b}$, hence $\left(H^{2} \Psi\right)^{i}=0$ on $\partial G$ and thus, using (2.11) again, $\left(H^{2} \Psi\right)^{e}=\Psi$ on $\partial G$. On the other hand, $M E^{2} \Psi$ is a solution of the exterior problem (2.1) with $\Phi=0$ on $\partial G$ satisfying the decay condition (2.3) (prescribe $a=0$ ) due to $\Psi_{M}=0$. By Lemma 2.1 this implies $E^{2} \Psi=\left(E^{2} \Psi\right)_{M}=$ const. in $G$, hence $\left(H^{2} \Psi\right)^{e}=\Psi=0$ on $\partial G$, as asserted. Thus the following theorem is proved.

THEOREM 2.2. Let $G \subseteq \mathbb{R}^{n}(n \geq 2)$ be an exterior domain with boundary $\partial G$ of class $C^{2}$, and let $\Phi \in C(\partial G)$ be given. In addition, if $n=2$, let $a \in \mathbb{R}$ be given. Then there is one and only one function $u \in C^{\infty}(G) \cap C(\bar{G})$ satisfying (2.1) in $G$ and the decay conditions (2.3). This solution admits in $G$ the following representation: if $n \geq 3$, then for any $\alpha$ with $0<\alpha \in \mathbb{R}$,

$$
u=D^{n} \Theta-\alpha E^{n} \Theta
$$

where $\Theta \in C(\partial G)$ is the uniquely determined solution of the boundary integral equation

$$
\Phi=-\frac{1}{2} \Theta+D^{n} \Theta-\alpha E^{n} \Theta \quad \text { on } \partial G .
$$


If $n=2$, then for any $\alpha, \beta$ with $0<\alpha \in \mathbb{R}, 0 \neq \beta \in \mathbb{R}$,

$$
u=-a \frac{\omega_{2}}{|\partial G|} E^{2} 1+D^{2} \Theta-\alpha E^{2} M \Theta-\beta \Theta_{M} .
$$

Here $a \in \mathbb{R}$ is the above-given constant appearing in (2.3), $E^{2} 1$ is the single-layer potential with constant density $\Psi=1$, and the projector $M$ is defined by $\Theta \mapsto M \Theta:=\Theta-\Theta{ }_{M}$ with the surface mean value $\Theta_{M}:=(1 /|\partial G|) \int_{\partial G} \Theta(y)$ do, where $\Theta \in C(\partial G)$ is the uniquely determined solution of the boundary integral equation

$$
\Phi+a \frac{\omega_{2}}{|\partial G|} E^{2} 1=-\frac{1}{2} \Theta+D^{2} \Theta-\alpha E^{2} M \Theta-\beta \Theta_{M} \quad \text { on } \partial G .
$$

3. Extension to homogeneous Sobolev spaces. The first theorem ensures the solvability of Laplace's equation (2.1) in the homogeneous spaces $S^{2, q}(G)$, defined by (1.5), in the case $n=2$.

THEOREM 3.1. Let $G \subseteq \mathbb{R}^{2}$ be an exterior domain with boundary $\partial G$ of class $C^{2}$, and let $\Phi \in W^{2-1 / q, q}(\partial G), 1<q<\infty$, and $a \in \mathbb{R}$ given. Then there is one and only one function $u \in S^{2, q}(G) \cap C^{\infty}(G)$ satisfying (2.1) and the decay conditions (2.3) for $n=2$.

Proof. Because for $n=2$ we have $(2-1 / q) q=2 q-1>1=n-1$, and Sobolev's Lemma [1] implies $\Phi \in C(\partial G)$, we can apply Theorem 2.2, obtaining a uniquely determined function $\Theta \in C(\partial G)$ satisfying the boundary integral equation (2.32). The function $u \in C^{\infty}(G) \cap C(\bar{G})$ defined by (2.31) fulfills (2.1) as well as the decay condition (2.3) for $n=2$, as shown above. Because the uniqueness has been established in Lemma 2.1, it remains to show $u \in S^{2, q}(G)$.

To do so, let $G_{r}:=G \cap B_{r}(0)$ as in the proof of Lemma 2.1. We obtain $u \in$ $W^{2-1 / q, q}\left(\partial G_{r}\right)$, because $u \in C^{\infty}(G)$ implies $u \in W^{2-1 / q, q}\left(\partial B_{r}\right)$ (see [7, page 238]), and because $u=\Phi \in W^{2-1 / q, q}(\partial G)$ on $\partial G$. Due to $u \in C^{\infty}\left(G_{r}\right) \cap C\left(\overline{G_{r}}\right)$ this implies $u \in$ $W^{2, q}\left(G_{r}\right)$ (see [7, page 232], which is based on [16, page 184]), and it remains to estimate the second-order derivatives of $u$ for $|x| \geq r$.

Using (2.31), we see that $\left|D_{k} D_{j} u(x)\right| \leq c_{r}|x|^{-2}$ for all $x$ with $|x| \geq r(k, j=1,2)$, which gives $D_{k} D_{j} u \in L^{q}\left(\mathbb{R}^{2} \backslash B_{r}\right)$ for all $1<q<\infty$. Thus $u \in S^{2, q}(G)$ as asserted and the theorem is proved.

The preceding arguments could be used for the case $n \geq 3$ and $q>n / 2$ as well, because, due to $(2-1 / q) q>n-1$, Sobolev's lemma [1] would imply $\Phi \in C(\partial G)$ as for $n=2$. The case $n \geq 3$ and $q \leq n / 2$, however, would not be included. Therefore, to prove the next theorem we use another approach which works for any $q$ with $1<q<\infty$ and any $n \geq 3$.

THEOREM 3.2. Let $G \subseteq \mathbb{R}^{n}(n \geq 3)$ be an exterior domain with boundary $\partial G$ of class $C^{2}$, and let $\Phi \in W^{2-1 / q, q}(\partial G), 1<q<\infty$, be given. Then there is one and only one function $u \in S^{2, q}(G) \cap C^{\infty}(G)$ satisfying (2.1) and the decay conditions (2.3) for $n \geq 3$.

Proof. To prove uniqueness, let $u=u^{1}-u^{2}$ be the difference of two solutions $u^{1}$ and $u^{2}$ with the required decay properties above. Define the bounded domain $G_{r}=$ $G \cap B_{r}(O)$, where $B_{r}(O) \subset \mathbb{R}^{n}$ denotes an open ball with center at zero and radius $r$ 
such that $\partial G \subset B_{r}(O)$. From the local regularity theory, we find $u \in W_{\text {loc }}^{2,2}(\bar{G})$. Thus in $G_{r}$ we may apply Green's first identity, and the uniqueness follows as in the proof of Lemma 2.1.

To prove existence, for $\Theta \in L^{q}(\partial G)$, we set

$$
T^{q_{\Theta}}:=D^{n} \Theta-\alpha E^{n} \Theta \quad(0<\alpha \in \mathbb{R}) .
$$

Then an easy calculation using Hölder's inequality shows that $T^{q}: L^{q}(\partial G) \rightarrow L^{q}(\partial G)$ is well defined and bounded. Now let $\Theta \in L^{q}(\partial G)$ be a solution of

$$
-\frac{1}{2} \Theta+T^{q} \Theta=0 .
$$

Then we find $\Theta \in L^{p}(\partial G)$ for some $p>n-1$. To see this we use the Hardy-LittlewoodSobolev inequality [19, page 119] obtaining in case of $1<q<n-1$ that $T^{q} \Theta \in L^{s}(\partial G)$ with

$$
\left\|T^{q} \Theta\right\|_{s, \partial G} \leq c_{q}\|\Theta\|_{q, \partial G} \quad\left(\frac{1}{s}=\frac{1}{q}-\frac{1}{n-1}\right) .
$$

Here we find $s>q$, and repeating this procedure a finite number of times, we obtain $\Theta \in L^{p}(\partial G)$ for some $p>n-1$. Next we show that $\Theta$ is bounded on $\partial G$. Since $\partial G \in C^{2}$ we have

$$
\begin{aligned}
|\Theta(x)| & =2\left|T^{q_{\Theta}}(x)\right| \leq c \int_{\partial G}|x-y|^{2-n}|\Theta(y)| d o_{y} \\
& \leq c\left(\int_{\partial G}|x-y|^{(2-n) p^{\prime}} d o_{y}\right)^{1 / p^{\prime}}\left(\int_{\partial G}|\Theta(y)|^{p} d o_{y}\right)^{1 / p},
\end{aligned}
$$

where the first integral on the right-hand side is finite due to $(n-2) p^{\prime}<n-1$ since $p>n-1\left(1 / p+1 / p^{\prime}=1\right)$. Now from the boundedness of $\Theta$ we obtain that $T^{q} \Theta$ is continuous on $\partial G$ (cf. [9, page 42] for $n=3$ ), and (3.2) implies the continuity of $\Theta$. Thus Theorem 2.2 implies

$$
\left\{\Theta \in L^{q}(\partial G) \mid-\frac{1}{2} \Theta+T^{q} \Theta=0\right\}=\left\{\Theta \in C(\partial G) \mid-\frac{1}{2} \Theta+D^{n} \Theta-\alpha E^{n} \Theta=0\right\}=\{0\} .
$$

Moreover, using a suitable cutoff procedure we obtain that the operator $T^{q}: L^{q}(\partial G) \rightarrow$ $L^{q}(\partial G)$ is compact, and applying the Fredholm alternative and the open mapping theorem we find that for any $\Phi \in L^{q}(\partial G)$ there is one and only one $\Theta \in L^{q}(\partial G)$ satisfying $\Phi=-(1 / 2) \Theta+T^{q} \Theta$ on $\partial G$ and the estimate

$$
\|\Theta\|_{q, \partial G} \leq c_{q}\left\|-\frac{1}{2} \Theta+T^{q} \Theta\right\|_{q, \partial G}=c_{q}\|\Phi\|_{q, \partial G} .
$$

Now we return to (2.1). Because of $\Phi \in W^{2-1 / q, q}(\partial G)$ there are functions $\Phi_{k} \in C^{2}(\partial G)$, $k \in \mathbb{N}$, such that

$$
\left\|\Phi_{k}-\Phi\right\|_{2-1 / q, q, \partial G} \longrightarrow 0 \quad \text { as } k \longrightarrow \infty
$$


Let $\Theta_{k}$ be the solution of the boundary integral equation (2.30) with $\Phi$ replaced by $\Phi_{k}$, corresponding to Theorem 2.2. Then this implies

$$
\Phi_{k}=-\frac{1}{2} \Theta_{k}+T^{q} \Theta_{k}
$$

Moreover, let $\Theta \in L^{q}(\partial G)$ denote the unique solution of

$$
\Phi=-\frac{1}{2} \Theta+T^{q} \Theta
$$

Then, using (3.6),

$$
\left\|\Theta-\Theta_{k}\right\|_{q, \partial G} \longrightarrow 0 \quad \text { as } k \longrightarrow 0 .
$$

For $x \in G$ and $k \in \mathbb{N}$ we define

$$
\begin{aligned}
u_{k}(x) & =D^{n} \Theta_{k}(x)-\alpha E^{n} \Theta_{k}(x), \\
u(x) & =D^{n} \Theta(x)-\alpha E^{n} \Theta(x) .
\end{aligned}
$$

Then, as shown above, $u_{k} \in C^{\infty}(G) \cap C(\bar{G})$ satisfies (2.1) with $\Phi=\Phi_{k}$, and, in particular, $u_{k} \in C^{2}\left(\partial G_{r}\right)$, where $G_{r}=G \cap B_{r}(0)$. Thus we conclude that $u_{k} \in W^{2, q}\left(G_{r}\right)$ with the following estimate:

$$
\left\|\left(u_{k}-u_{l}\right)\right\|_{2, q, G_{r}} \leq c_{q, r}\left(\left\|u_{k}-u_{l}\right\|_{2-1 / q, q, \partial G}+\left\|u_{k}-u_{l}\right\|_{2-1 / q, q, \partial B_{r}}\right),
$$

(see [6, page 340], which is based on [16, page 184]). Because $u_{k}-u_{l}=\Phi_{k}-\Phi_{l}$ on $\partial G$, the first term on the right-hand side of (3.12) tends to zero as $k, l \rightarrow \infty$. For the second term we find

$$
\left\|u_{k}-u_{l}\right\|_{2-1 / q, q, \partial B_{r}} \leq c_{q, r}\left(\left\|\Theta_{k}-\Theta_{l}\right\|_{q, \partial G}\right)
$$

(cf. [7, page 238]). Thus, due to (3.10), $u_{k}$ is a Cauchy sequence in $W^{2, q}\left(G_{r}\right)$. Moreover, Hölder's inequality together with (3.10) shows that for any $x \in G$ we have $u_{k}(x) \rightarrow$ $u(x)$ as $k \rightarrow \infty$, hence $u \in W^{2, q}\left(G_{r}\right)$ with $\left\|u-u_{k}\right\|_{2, q, G_{r}} \rightarrow 0$ as $k \rightarrow \infty$. This implies $\left\|u-u_{k}\right\|_{2-1 / q, q, \partial G} \rightarrow 0$ as $k \rightarrow \infty$, and because $u_{k}=\Phi_{k}$ on $\partial G$, (3.7) yields $u=\Phi \in$ $W^{2-1 / q, q}(\partial G)$ on $\partial G$. Because $u \in C^{\infty}(G)$ with $\Delta u=0$ in $G$, and because $u$ satisfies the decay properties (2.3) for $n \geq 3$, the second-order derivatives $D_{k} D_{j} u(x)(k, j=$ $1, \ldots, n)$ for all $x$ with $|x| \geq r$ can be estimated as in the case $n=2$ (see the proof of Theorem 3.1). Thus $u \in S^{2, q}(G)$ and the theorem is proved.

The next theorem ensures the solvability of Poisson's equation (1.1) in the spaces $S^{2, q}(G)$, defined by (1.5).

THEOREM 3.3. Let $G \subset \mathbb{R}^{n}(n \geq 2)$ be an exterior domain with boundary $\partial G$ of class $C^{2}$, and let $1<q<\infty$. Then for every $f \in L^{q}(G)$ and $\Phi \in W^{2-1 / q, q}(\partial G)$ there exists some $u \in S^{2, q}(G)$ satisfying the Poisson equation (1.1) in $G$.

Proof. Setting $f=0$ in $\mathbb{R}^{n} \backslash \bar{G}$ we obtain some function $\tilde{f} \in L^{q}\left(\mathbb{R}^{n}\right)$ with $\tilde{f}_{\mid G}=f$ in $G$. Let $\tilde{f}_{i} \in C_{0}^{\infty}\left(\mathbb{R}^{n}\right)$ denote a sequence such that $\tilde{f}_{i} \rightarrow \tilde{f}$ in $L^{q}\left(\mathbb{R}^{n}\right)$ as $i \rightarrow \infty$. Consider 
now for fixed $i$ the equation $-\Delta \tilde{u}_{i}=\tilde{f}_{i}$ in $\mathbb{R}^{n}$. We can solve it by convolution with $E_{n}$ (see (2.2)), obtaining in $x \in \mathbb{R}^{n}$ the representation

$$
\tilde{u}_{i}(x)=\left(E_{n} * \tilde{f}_{i}\right)(x)=\int_{\mathbb{R}^{n}} E_{n}(x-y) \tilde{f}_{i}(y) d y .
$$

Moreover, by the theorem of Calderón and Zygmund [4], for the second-order derivatives we obtain the estimate $\left\|\nabla^{2} \tilde{u}_{i}\right\|_{q} \leq c\left\|\tilde{f}_{i}\right\|_{q}$ with some constant $c$ independent of $i \in \mathbb{N}$, which implies $\left\|\nabla^{2}\left(\tilde{u}_{i}-\tilde{u}_{k}\right)\right\|_{q} \rightarrow 0$ as $i, k \rightarrow \infty$.

Next consider a sequence of open balls $\left(B_{j}\right)_{j}$ with $B_{j} \subset B_{j+1}$ and $\bigcup_{j=1}^{\infty} B_{j}=\mathbb{R}^{n}$. We define the space

$$
\mathbb{P}=\left\{P: x \rightarrow P(x)=a+b \cdot x \mid b, x \in \mathbb{R}^{n}, a \in \mathbb{R}\right\}
$$

of linear functions $P: \mathbb{R}^{n} \rightarrow \mathbb{R}$. Then by the generalized Poincaré inequality (cf. [12, page $22]$ or [14, page 112]) we obtain for every $v \in S^{2, q}\left(\mathbb{R}^{n}\right)$ the estimate

$$
\|v\|_{L^{q\left(B_{j}\right) / \mathbb{P}}}:=\inf _{P \in \mathbb{P}}\|v+P\|_{L^{q\left(B_{j}\right)}} \leq c_{j}\left\|\nabla^{2} v\right\|_{L^{q}\left(B_{j}\right)} n^{2}
$$

with some constants $c_{j}>0$. Because $\tilde{u}_{i} \in S^{2, q}\left(\mathbb{R}^{n}\right)$, we conclude that $\left(\tilde{u}_{i}\right)_{i}$ is a Cauchy sequence with respect to the norm $\|\cdot\|_{L^{q}\left(B_{1}\right) / \mathbb{P}}$ on the left-hand side of (3.16) for fixed $j=1$. This implies the existence of linear functions $P_{i} \in \mathbb{P}$ such that $\left(\tilde{u}_{i}+P_{i}\right)_{i}$ is Cauchy sequence in $L^{q}\left(B_{1}\right)$. Repeating this argument now for $j=2$, there exist linear functions $Q_{i} \in \mathbb{P}$ such that $\tilde{u}_{i}+Q_{i}$ is a Cauchy sequence in $L^{q}\left(B_{2}\right)$, hence in $L^{q}\left(B_{1}\right)$, and using the representation

$$
P_{i}(x)=\alpha_{i}+\beta_{i} \cdot x, \quad Q_{i}(x)=\gamma_{i}+\delta_{i} \cdot x,
$$

we obtain that $\left(\alpha_{i}-\gamma_{i}\right)_{i}$ and $\left(\beta_{i}-\delta_{i}\right)_{i}$ are Cauchy sequences in $\mathbb{R}$ and in $\mathbb{R}^{n}$, respectively. From this we find that $\left(P_{i}-Q_{i}\right)_{i}$ is a Cauchy sequence in $L^{q}\left(B_{2}\right)$, and thus also $\left(\tilde{u}_{i}+\right.$ $\left.P_{i}\right)_{i}=\left(\tilde{u}_{i}+Q_{i}\right)_{i}+\left(P_{i}-Q_{i}\right)_{i}$. Repeating this procedure it follows that $\left(\tilde{u}_{i}+P_{i}\right)_{i}$ is a Cauchy sequence in $L^{q}\left(B_{j}\right)$ for all $j=1,2, \ldots$. Thus we can find some $\tilde{u} \in S^{2, q}\left(\mathbb{R}^{n}\right)$ such that

$$
\left(\tilde{u}_{i}+P_{i}\right) \longrightarrow \tilde{u} \quad \text { in } L_{\mathrm{loc}}^{q}\left(\mathbb{R}^{n}\right), \quad\left\|\nabla^{2}\left(\tilde{u}-\tilde{u}_{i}\right)\right\|_{q, \mathbb{R}^{n}} \longrightarrow 0 \quad \text { as } i \longrightarrow \infty
$$

Moreover, $\tilde{u}$ satisfies $-\Delta \tilde{u}=\tilde{f}$ in $\mathbb{R}^{n}$ and the estimate $\left\|\nabla^{2} \tilde{u}\right\|_{q} \leq c\|\tilde{f}\|_{q}$. Since $\tilde{u} \in$ $W_{\text {loc }}^{2, q}\left(\mathbb{R}^{n}\right)$ we conclude from the usual trace theorem [1, page 217] that $\tilde{u}_{\mid \partial G} \in$ $W^{2-1 / q, q}(\partial G)$. Following Lemma 2.1 there is a function $w \in S^{2, q}(G)$ satisfying the equations

$$
-\Delta w=0 \quad \text { in } G, \quad w_{\mid \partial G}=\tilde{u}_{\mid \partial G}-\Phi,
$$

where $\Phi \in W^{2-1 / q, q}(\partial G)$ is the prescribed boundary value. Now setting $u=\tilde{u}_{\mid G}-w$, we obtain the desired solution and the theorem is proved. 
Because functions $u \in S^{2, q}(G)$ have no suitable decay properties at infinity, in general we cannot expect uniqueness for the solution of (1.1) constructed in Theorem 3.1. Thus we consider in $G$ the homogeneous equations and define the nullspace of (1.1) by

$$
N_{q}(G)=\left\{u \in S^{2, q}(G) \mid-\Delta u=0 \text { in } G, u_{\mid \partial G}=0\right\} .
$$

THEOREM 3.4. Let $G \subset \mathbb{R}^{n}(n \geq 2)$ be an exterior domain with boundary $\partial G$ of class $C^{2}$, and let $1<q<\infty$. Then for the dimension $\operatorname{dim} N_{q}(G)$ of the nullspace defined in (3.20), $\operatorname{dim} N_{q}(G)=n+1$ independent of $q$.

Proof. Consider the space $\mathbb{P}$ of linear functions defined in (3.15). Because for every $P \in \mathbb{P}$ we have $P(x)=a+b \cdot x$ with some $a \in \mathbb{R}$ and some vector $b \in \mathbb{R}^{n}$, we find $\operatorname{dim} \mathbb{P}=n+1$. Let $u^{P}$ denote the uniquely determined solution of the equation

$$
-\Delta u=0, \quad u_{\mid \partial G}=-P_{\mid \partial G}
$$

with $P \in \mathbb{P}$, according to Lemma 2.1. Here in the case $n=2$ we require

$$
u(x)-a \ln |x|=O(1) \quad \text { as }|x| \longrightarrow \infty,
$$

where the constant $a$ is chosen from $P(x)=a+b \cdot x$. Setting

$$
M_{\mathcal{q}}(G)=\left\{u^{P}+P_{\mid \bar{G}} \mid P \in \mathbb{P}\right\}
$$

we obtain $M_{q}(G) \subset N_{q}(G)$, obviously. Furthermore, we have $\operatorname{dim} M_{q}(G)=\operatorname{dim} \mathbb{P}=n+1$, which can be shown as follows. Let $P(x)=a+b \cdot x$ and let $u^{P}+P_{\mid \bar{G}}=0$ in $\bar{G}$. Then from the decay properties of $u^{P}$ and $\nabla u^{P}$ established in Lemma 2.1 we find $a=0$ and $b=0$, hence $P=0$. Here in the case $n=2$ we obtain $a=0$ due to the special choice of the number $a$ in (3.22). Together with the uniqueness statement in Lemma 2.1, this means that, if $B$ is a basis of $\mathbb{P}$, then

$$
B_{q}(G)=\left\{u^{P}+P_{\mid \bar{G}} \mid P \in B\right\}
$$

is a basis of $M_{q}(G)$. Thus it remains to show that

$$
N_{q}(G) \subset M_{q}(G)
$$

To do so, we first determine the nullspace

$$
N_{q}\left(\mathbb{R}^{n}\right)=\left\{u \mid u \in S^{2, q}\left(\mathbb{R}^{n}\right) \text { with }-\Delta u=0 \text { in } \mathbb{R}^{n}\right\}
$$

From $\Delta u=0$, hence $\Delta \nabla^{2} u=0$ with $D_{j k}^{2} u \in L^{q}\left(\mathbb{R}^{n}\right)(j, k=1, \ldots, n)$ we obtain $\nabla^{2} u=0$ in $\mathbb{R}^{n}$, which implies $u=P$ for some $P \in \mathbb{P}$. Thus we have shown that

$$
N_{q}\left(\mathbb{R}^{n}\right)=\mathbb{P} .
$$


Now let $u \in N_{q}(G)$. We extend $u$ on the whole space obtaining a function $\tilde{u} \in S^{2, q}\left(\mathbb{R}^{n}\right)$ with $\tilde{u}_{\mid \bar{G}}=u$ [1, page 83]. Moreover, this function satisfies in $\mathbb{R}^{n}$ the identity $-\Delta \tilde{u}=\tilde{f} \in$ $L^{q}\left(\mathbb{R}^{n}\right)$, where the function $\tilde{f}$ has a compact support in the bounded domain $\mathbb{R}^{n} \backslash \bar{G}$. Consider the equation

$$
-\Delta w=\tilde{f} \text { in } \mathbb{R}^{n}
$$

Again, it can be solved by convolution with the fundamental solution $E_{n}$ of the Laplacian: we obtain $w=E_{n} * \tilde{f}$ in $\mathbb{R}^{n}$ and the Calderón-Zygmund theorem implies $D_{j k}^{2} w \in$ $L^{r}\left(\mathbb{R}^{n}\right)$ for all $1<r \leq q(j, k=1, \ldots, n)$. Here we used $\tilde{f} \in L^{r}\left(\mathbb{R}^{n}\right)^{n}$ for all $1<r \leq q$ due to its compact support. Now using a well-known estimate of Hardy-Littlewood-Sobolevtype [2, page 242] we find $w \in L^{s}\left(\mathbb{R}^{n}\right)$ for some $s \geq q$, hence $w \in L_{\text {loc }}^{s}\left(\mathbb{R}^{n}\right) \subset L_{\text {loc }}^{q}\left(\mathbb{R}^{n}\right)$. Thus we have constructed some solution $w$ of (3.28) such that $w \in S^{2, q}\left(\mathbb{R}^{n}\right)$. Setting $W=\tilde{u}-w$, we obtain $W \in N_{q}\left(\mathbb{R}^{n}\right)$, and (3.27) leads to $\tilde{u}=w+P$ for some $P \in \mathbb{P}$. Because $\tilde{u}_{\mid \partial G}=0$ and since $\tilde{u}_{\mid \bar{G}}=u$, we find $u \in M_{\mathcal{q}}(G)$, which proves (3.25) and thus the theorem.

\section{REFERENCES}

[1] R. A. Adams, Sobolev Spaces, Academic Press, New York, 1975.

[2] L. Bers, F. John, and M. Schechter, Partial Differential Equations, American Mathematical Society, Rhode Island, 1979.

[3] H. Brakhage and P. Werner, Über das Dirichlet'sche Außenraumproblem für die Helmholtz'sche Schwingungsgleichung, Arch. Math. 16 (1965), 325-329 (German).

[4] A. P. Calderón and A. Zygmund, On singular integrals, Amer. J. Math. 78 (1956), 289-309.

[5] J. Deny and J. L. Lions, Les espaces du type de Beppo Levi, Ann. Inst. Fourier (Grenoble) 5 (1953/1954), 305-370 (French).

[6] P. Deuring, The resolvent problem for the Stokes system in exterior domains: an elementary approach, Math. Methods Appl. Sci. 13 (1990), no. 4, 335-349.

[7] P. Deuring, W. von Wahl, and P. Weidemaier, Das lineare Stokes-System in $\mathbf{R}^{3}$. I. Vorlesungen über das Innenraumproblem, Bayreuth. Math. Schr. 27 (1988), 1-252 (German).

[8] J. Giroire, Etude de quelques problemes aux limites exterieurs et resolution par equations integrals, Thèse de doctorat d'etat es sciences mathematiques, Paris 6: Université Pierre et Marie Curie, Paris, 1987.

[9] N. M. Günter, Die Potentialtheorie und ihre Anwendung auf Grundaufgaben der mathematischen Physik, B. G. Teubner Verlagsgesellschaft, Leipzig, 1957.

[10] G. C. Hsiao and R. Kress, On an integral equation for the two-dimensional exterior Stokes problem, Appl. Numer. Math. 1 (1985), no. 1, 77-93.

[11] R. Leis, Zur Eindeutigkeit der Randwertaufgaben der Helmholtz'schen Schwingungsgleichung, Math. Z. 85 (1964), 141-153 (German).

[12] V. G. Maz'ja, Sobolev Spaces, Springer Series in Soviet Mathematics, Springer-Verlag, Berlin, 1985.

[13] R. C. McOwen, The behavior of the Laplacian on weighted Sobolev spaces, Comm. Pure Appl. Math. 32 (1979), no. 6, 783-795.

[14] J. Nečas, Les Méthodes Directes en Théorie des Équations Elliptiques, Academia, Prague, 1967.

[15] J. Saranen and K. J. Witsch, Exterior boundary value problems for elliptic equations, Ann. Acad. Sci. Fenn. Ser. A I Math. 8 (1983), no. 1, 3-42.

[16] C. G. Simader, On Dirichlet's Boundary Value Problem, Springer-Verlag, Berlin, 1972. 
[17] C. G. Simader and H. Sohr, The Dirichlet Problem for the Laplacian in Bounded and Unbounded Domains, Pitman Research Notes in Mathematics Series, vol. 360, Longman, Harlow, 1996.

[18] W. I. Smirnow, Lehrgang der höheren Mathematik. Teil IV [Course in Higher Mathematics. Part IV], 10th ed., Hochschulbücher für Mathematik, vol. 5, VEB Deutscher Verlag der Wissenschaften, Berlin, 1982, translated from the third Russian edition by Christa Berg and Lothar Berg.

[19] E. M. Stein, Singular Integrals and Differentiability Properties of Functions, Princeton Mathematical Series, no. 30, Princeton University Press, New Jersey, 1970.

[20] W. Varnhorn, The Poisson equation with weights in exterior domains of $\mathbf{R}^{n}$, Appl. Anal. 43 (1992), no. 1-2, 135-145.

Tatiana Samrowski: Fachbereich 17 Mathematik/Informatik, Universität Kassel, Heinrich-PlettStr. 40, 34109 Kassel, Germany

E-mail address: samrowski@mathematik. uni-kasse1. de

Werner Varnhorn: Fachbereich 17 Mathematik/Informatik, Universität Kassel, Heinrich-PlettStr. 40, 34109 Kassel, Germany

E-mail address: varnhorn@mathematik.uni-kasse1.de 


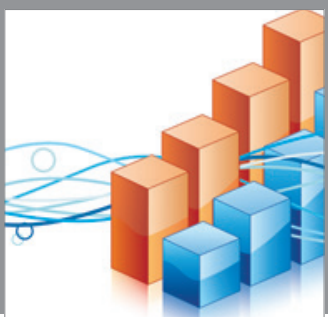

Advances in

Operations Research

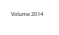

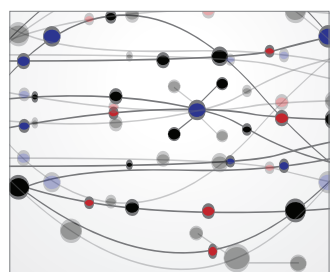

\section{The Scientific} World Journal
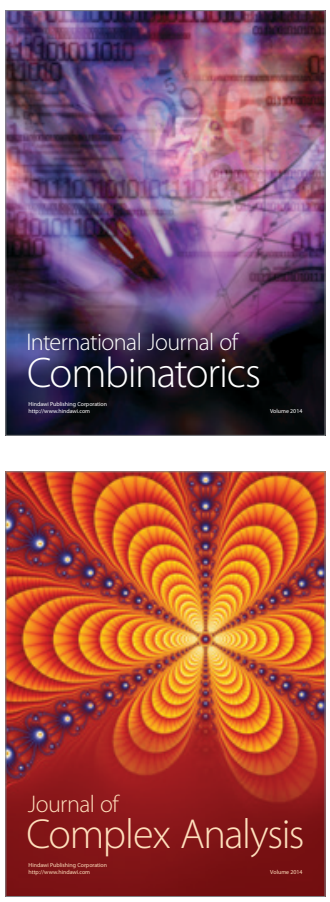

International Journal of

Mathematics and

Mathematical

Sciences
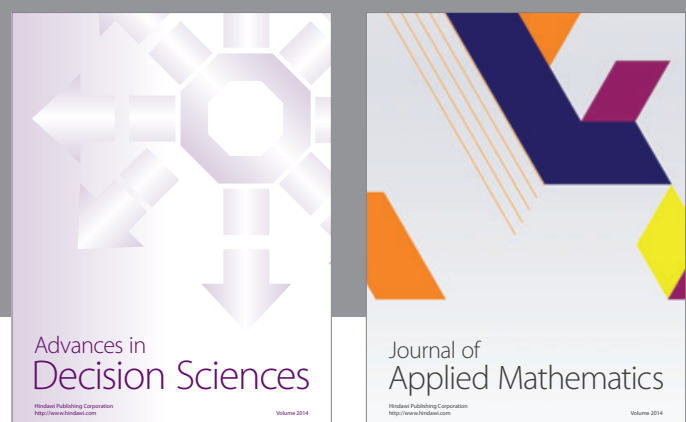

Journal of

Applied Mathematics
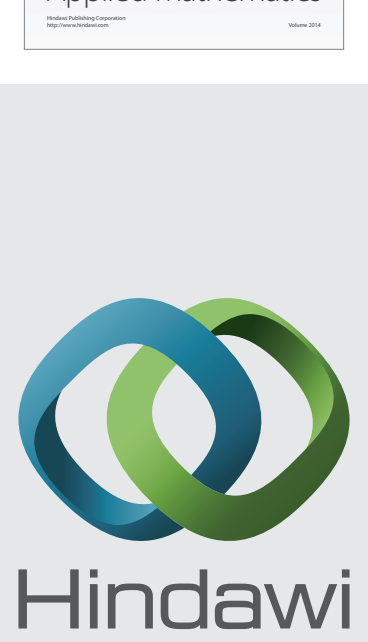

Submit your manuscripts at http://www.hindawi.com
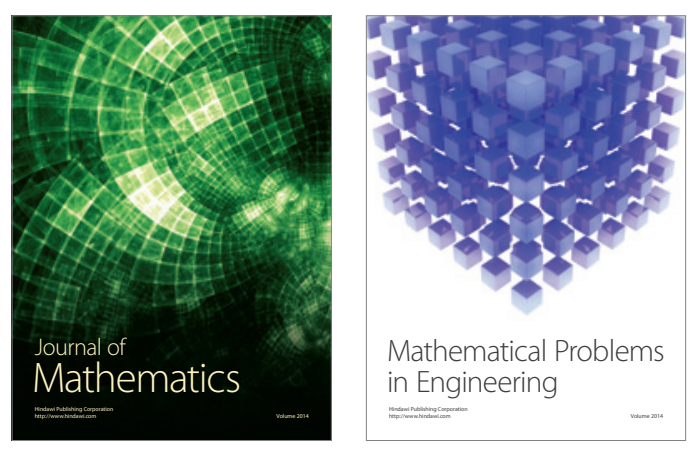

Mathematical Problems in Engineering
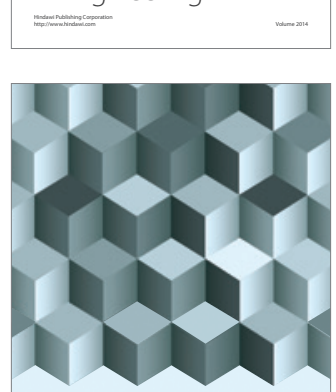

Journal of

Function Spaces
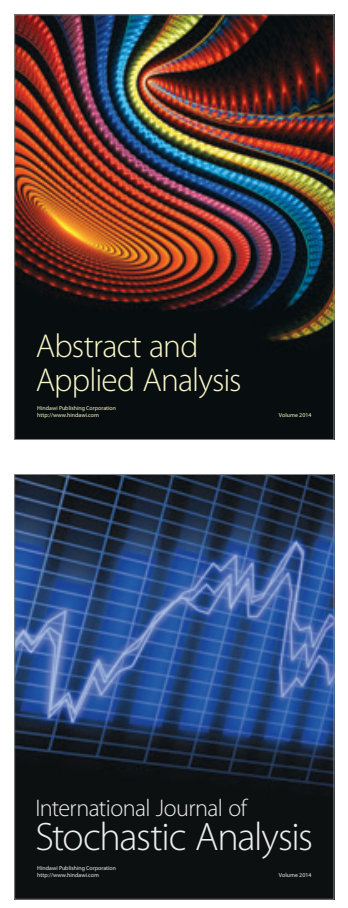

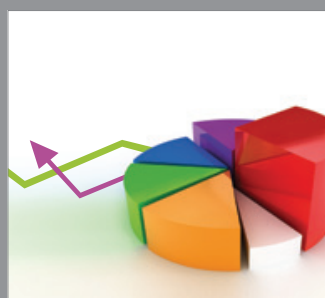

ournal of

Probability and Statistics

Promensencen
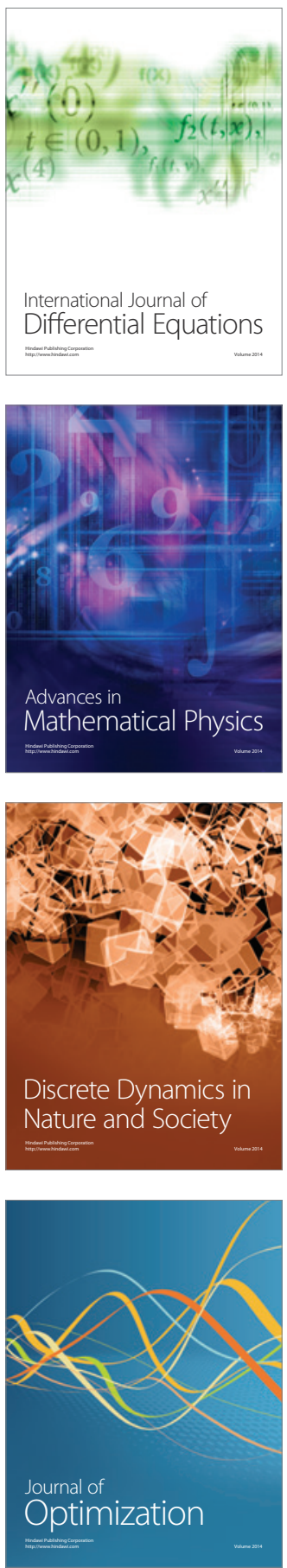